\title{
Aplicação terapêutica da fibrina rica em plaqueta (L-PRF) em paciente submetido a exodontia de terceiros molares
}

Therapeutic application of platelet-rich fibrin (L-PRF) in patient submitted to third molar exodonty

Aplicación terapéutica de fibrina rica en plaquetas (L-PRF) en paciente sometido a tercer exodoncia molar

Marcos Antônio De Araújo Júnior

ORCID: https://orcid.org/0000-0003-0004-0056 Centro Universitário Fluminense, Brasil E-mail: urjmrcs@gmail.com

Maria Cecília Simões Riscado De Castro

ORCID: https://orcid.org/0000-0003-3846-8074 Centro Universitário Fluminense, Brasil E-mail: cecilia riscado@hotmail.com

Larissa Santos Magalhães da Silva ORCID: https://orcid.org/0000-0002-1634-5818 Centro Universitário Fluminense, Brasil E-mail: larissamagalhaes227@gmail.com

Lara Santos da Silva

ORCID: https://orcid.org/0000-0003-2391-5118 Centro Universitário Fluminense, Brasil E-mail: larasantos1063@hotmail.com

Camila Soares Corrêa

ORCID: https://orcid.org/0000-0001-7707-9560 Centro Universitário Fluminense, Brasil E-mail: milasoarescorrea@gmail.com

Hugo Leonardo Matias Nahmias ORCID: https://orcid.org/0000-0003-4083-3261 Centro Universitário Fluminense, Brasil E-mail: hugonahmiasph@gmail.com

Victor Paes Dias Goncalves

ORCID: https://orcid.org/0000-0001-6469-5735 Associação Brasileira de Odontologia Regional Campos, Brasil E-mail: victor.dias.paes@gmail.com

Melissa Paes Petrucci

ORCID: https://orcid.org/0000-0002-5165-8588 Centro Universitário Fluminense, Brasil E-mail: melpaesp@gmail.com

\begin{abstract}
Resumo
A exodontia dos terceiros molares é um dos procedimentos rotineiros mais realizados pelos cirurgiões dentistas e que apresenta frequentemente um grande desconforto pós-operatório. A fibrina rica em plaquetas é uma alternativa para minimizar os sintomas pós operatórios, exercendo resultados positivos no processo de cicatrização, além de se tratar de um atrativo biomaterial a ser empregado na prática clínica. Buscamos com o presente estudo, descrever um relato de caso clínico com a utilização do L-PRF após exodontia de $3^{\circ}$ molar inferior. Concluímos que o uso do L-PRF em cirurgias de terceiros molares é uma alternativa capaz de proporcionar um pós-operatório menos invasivo ao paciente, que está ao alcance do cirurgião-dentista, por apresentar um baixo custo e facilidade de manipulação.
\end{abstract}

Palavras-chave: Cirurgia oral; Cirurgia do $3^{\circ}$ molar; Cura.

\begin{abstract}
Third molar extraction is one of the most routine procedures performed by dentists and often presents great postoperative discomfort. Platelet-rich fibrin is an alternative to minimize postoperative symptoms, exerting positive results in the healing process, in addition to being an attractive biomaterial to be used in clinical practice. With the present study, we seek to describe a clinical case report with the use of the L-PRF after extraction of the 3rd mandibular molar. We conclude that the use of L-PRF in third molar surgeries is an alternative capable of providing a less invasive postoperative period to the patient, which is within the reach of dentists, due to its low cost and ease of handling.

Keywords: Oral Surgery; 3rd Molar Surgery; Healing.
\end{abstract}




\section{Resumen}

La extracción del tercer molar es uno de los procedimientos más rutinarios que realizan los odontólogos y muchas veces presenta un gran malestar postoperatorio. La fibrina rica en plaquetas es una alternativa para minimizar los síntomas postoperatorios, ejerciendo resultados positivos en el proceso de cicatrización, además de ser un biomaterial atractivo para ser utilizado en la práctica clínica. Con el presente estudio buscamos describir el reporte de un caso clínico con el uso del L-PRF luego de la extracción del 3er molar mandibular. Concluimos que el uso de L-PRF en cirugías de terceros molares es una alternativa capaz de brindar un postoperatorio menos invasivo al paciente, que está al alcance de los odontólogos, por su bajo costo y facilidad de manejo.

Palabras clave: Cirugía bucal; Cirugía de 3er molar; Curación.

\section{Introdução}

Com o decorrer dos anos e as mudanças dos hábitos alimentares, a ausência de elementos dentários deixou de ser vista como casos isolados. Entretanto, mesmo considerando a agenesia de terceiros molares em grande prevalência, a exodontia desse elemento ainda é um procedimento frequente no cotidiano do cirurgião dentista (CD), quer seja para fins de tratamentos dos sintomas, por razões profiláticas ou até mesmo ortodônticas.

A remoção cirúrgica do terceiro molar, embora seja um procedimento relativamente comum, é uma operação invasiva e comumente realizada em pessoas jovens e saudáveis, as quais, na maioria das vezes, não possui experiência com cirurgias desse tipo, além disso, os terceiros molares são potencialmente capazes de causar transtornos e prejuízos à saúde bucal do indivíduo, risco que justifica a indicação para exodontia (Uyanık et al., 2015; Benedito et al., 2021).

Os sintomas pós-operatórios podem gerar um mal-estar demasiado, tendo em vista que a inflamação aguda é constituída por um conjunto de sintomas como dor violenta, edema e dificuldade na abertura de boca (Santos Gonçalves et al., 2020; Silva Conceição et al., 2020; Gonçalves et al., 2021). Dessa forma, gera dificuldade da fala, mastigação e higienização do local. Na esfera da cirurgia regeneradora, o aumento e a incessante busca por recursos rápidos e efetivos capazes de aprimorar a reparação de tecidos têm oferecido nos últimos anos, estímulos sobre a produção de matérias primas e técnicas dispostas a simplificar a complexa etapa cicatricial iniciada após o ato cirúrgico (Costa et al. 2021).

Choukroun et al., (2006) sugerem uma alternativa de biomaterial autólogo, leuco-plaquetário, de fácil manipulação e baixos riscos de infecção cruzada, denominado de Fibrina Rica em Plaquetas (L-PRF). As aplicações clínicas destacadas com o uso do L-PRF são concebidas em quatro episódios imprescindíveis para a cicatrização, compondo-se pela angiogênese, controle imunológico, aproveitamento de células-tronco circulantes e recobrimento de ferida por epitélio (Peralvo et al., 2017; Favero et al., 2017). Esses episódios são importantes para a obtenção de uma cicatrização de tecidos de forma mais rápida devido ao desenvolvimento eficaz da neovascularização, acelerando o fechamento da ferida com rápida remodelação do tecido cicatricial e escassez quase total desses eventos de infecção (Mourão et al., 2015; Campos et al., 2021). Desta forma, o L-PRF é capaz de promover uma cicatrização acelerada devido ao desenvolvimento de uma neovascularização eficaz, controle imunológico, recrutamento das células-tronco circundantes que levará ao fechamento acelerado da ferida com remodelação rápida do tecido cicatricial, aspectos altamente específicos para a cicatrização, além de diminuir da resposta dolorosa à lesão (Dohan et al., 2006).

\section{Metodologia}

Este trabalho trata se de um estudo de caso único, pois possui a intenção de descrever um relato de caso clínico com a utilização do L-PRF após exodontia de $3^{\circ}$ molar inferior. Sendo tratado em um estudo qualitativo, verificando e possibilitando a resolução das problemáticas solicitadas pela paciente (Pereira et al., 2018). Este estudo foi realizado através uma anamnese criteriosa onde o paciente de 23 anos, procurou a clínica da disciplina de cirurgia da $\mathrm{ABO} / \mathrm{CAMPOS}$, relatando sintomatologia dolorosa na região do terceiro molar inferior com exame radiográfico panorâmico. Os 
responsáveis pela paciente examinanda assinaram o Termo de Consentimento Livre e Esclarecido (TCLE), autorizando a realização de registros de fotografias.

Figura 1: Imagem Panorâmica.

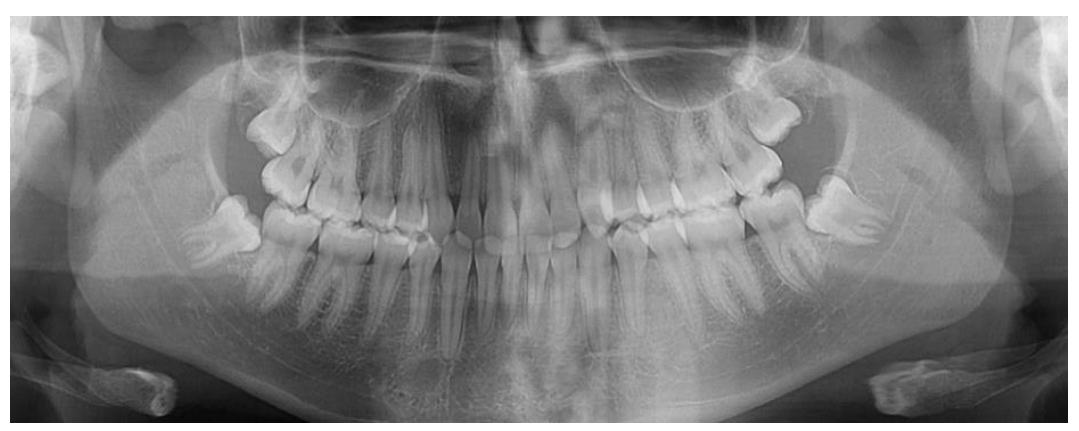

Fonte: Autores.

\section{Relato de Caso}

Para a realização da cirurgia não será feita medicação pré-operatória. Anteriormente ao início do procedimento será feita a antissepsia extraoral utilizando digluconato de clorexidina a $2 \%$, e intraoral utilizando digluconato de clorexidina a $0,12 \%$ na forma de bochecho. Em sequência será realizada aposição dos campos cirúrgicos estéreis e anestesia tópica com Benzocaína $20 \%$, aplicada em mucosa seca por 1 minuto. A técnica anestésica utilizada será do tipo bloqueio regional do nervo alveolar póstero-inferior, nervo bucal e nervo lingual, a solução anestésica de escolha será lidocaína 2\% com epinefrina 1:100.000 (DFL).

A cirurgia será realizada unilateralmente em uma das regiões de terceiro molar inferior (elemento 38 ou 48), a escolha será feita previamente ao procedimento após análise dos exames radiográficos de acordo com o sítio cirúrgico que se adapte melhor a aplicação da técnica de L-PRF, a decisão será tomada em conjunto com a equipe participante levando em consideração o planejamento cirúrgico. Será executada a incisão avelanal com lâmina de aço $\left(n^{\circ} 11\right)$, seguida de descolamento mucoperiósteo que será feito com descolador de molt (Figura 2), osteotomia com broca carbide cirúrgica esférica (Figura 3), odontosecção com broca Zecrya(Figura 4) e luxação do elemento com alavanca Seldin reta $\left(n^{\circ} 2\right)$, para obtenção da exérese (Figura 5).Colocação do L-PRF no alvéolo (Figura 5).

Figura 2: Incisão Avelanal.

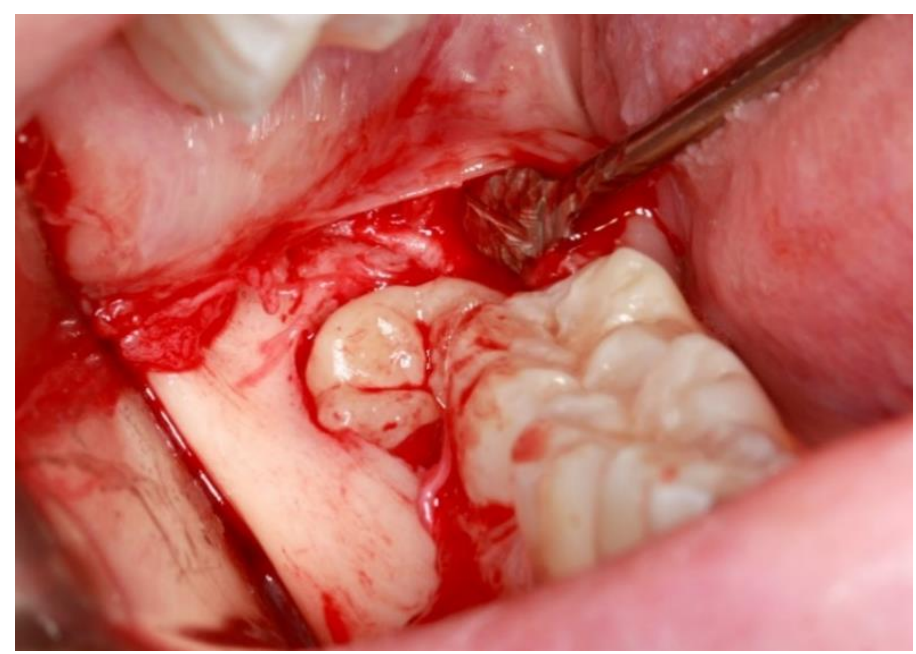

Fonte: Autores. 
Figura 3: Ostetomia.

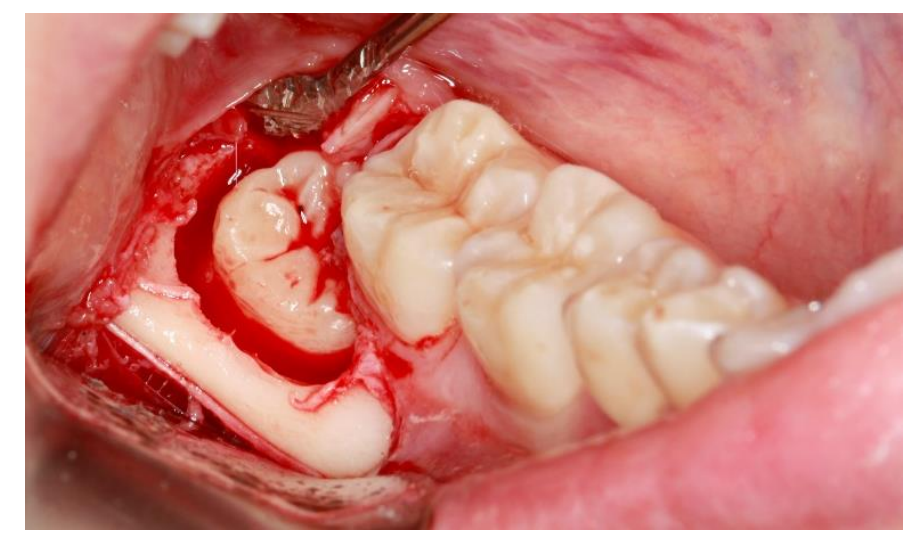

Fonte: Autores.

Figura 4: Odontosecção.

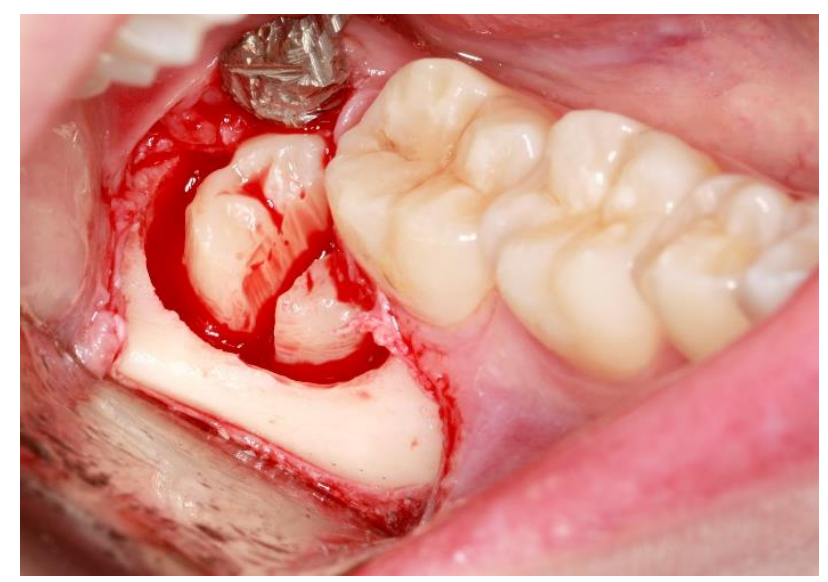

Fonte: Autores.

Figura 5: L-PRF no alvéolo.

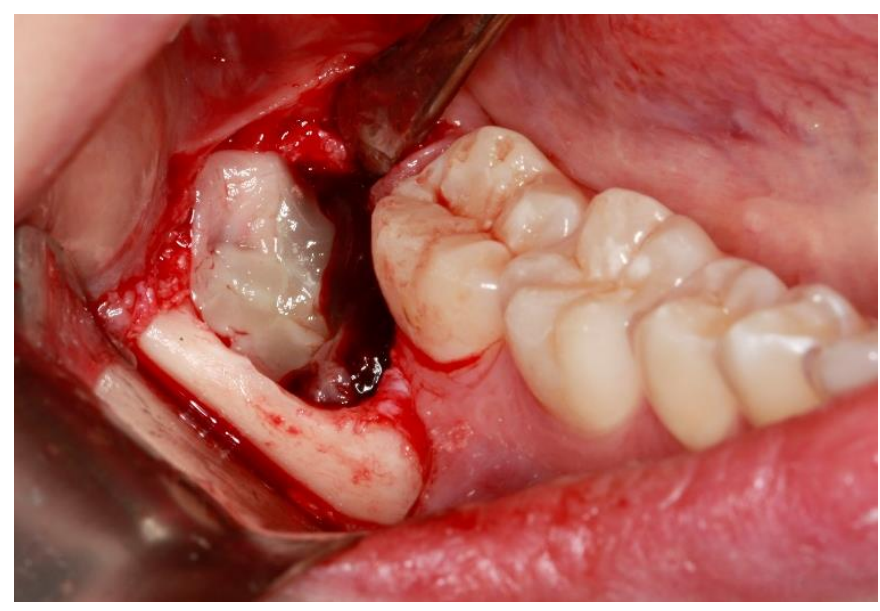

Fonte: Autores.

Após curetagem, será realizada irrigação abundante com soro fisiológico $0,9 \%$ no interior do alvéolo a fim de se remover quaisquer tipos de resíduos e em sequência reposicionamento/sutura do retalho com pontos simples (Figura 6), usando fio de nylon 3-0 não absorvível e agulha circular 3/8 de 2,0 cm (BC Suture). A sutura será removida após 7 dias (Figura 7). 
Figura 6: Sutura aspecto final.

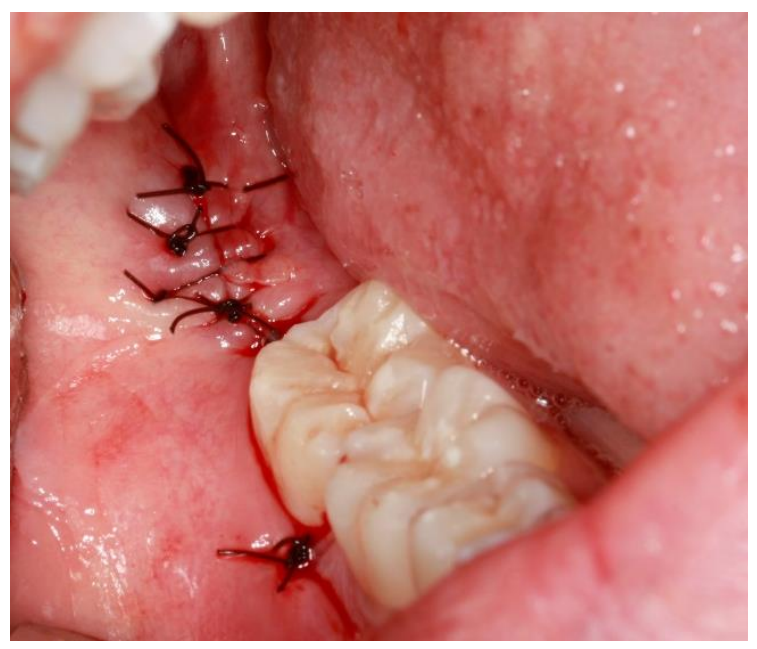

Fonte: Autores.

Figura 7: Aspecto após 7 dias, após remoção de sutura.

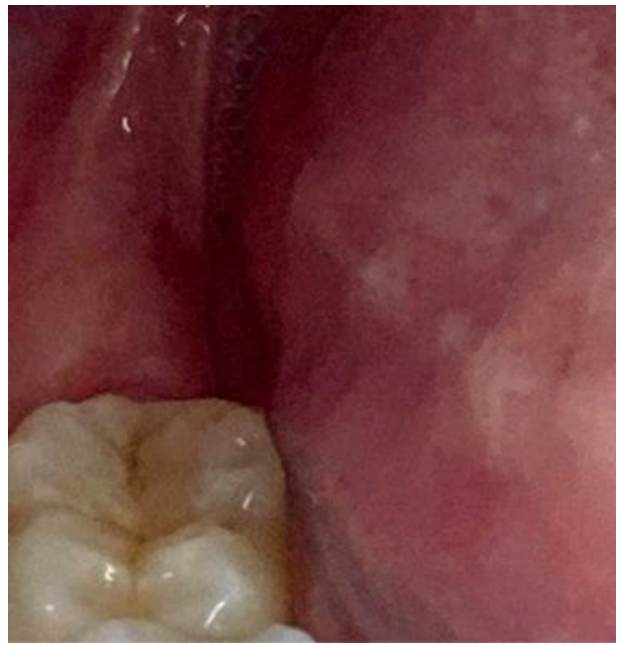

Fonte: Autores.

Em detrimento ao procedimento executado será realizada como medicação pós-operatória: Cetorolaco de trometamol 10mg via sublingual de imediato após o término da cirurgia e após a paciente foi orientada a tomar apenas em caso de dor.

O conforto pós operatório do paciente, foi utilizado uma escala analógica visual da dor, o nível de dor foi avaliado em diversos intervalos até a remoção dos pontos dentro de um período de 7 dias junto com a quantidade de analgésico.

\section{Resultados e Discussão}

Em relação a escala analógica de dor a paciente relatou escores 5 nas primeiras 24 horas, escore 4 nas primeiras 48 horas, escore 3 nas primeiras 72 horas, escore 1 com 7 dias. Sobre a utilização de analgésicos, apenas um analgésico nas primeiras 24 horas, um analgésico e zero analgésico em diante até o dia da remoção dos pontos.

Esse resultado foi similar ao encontrado no trabalho de Caymaz e Uyanik (2018) onde foi realizado cirurgias em 27 pacientes com terceiro molar inferior impactado bilateralmente, que operaram cirurgicamente em momentos diferentes em 2 grupos: A-PRF e L-PRF, onde o L-PRF foi superior na diminuição da escala da dor e no número de analgésicos utilizados. 
A utilização de bioativos para fins terapêuticos e a compreensão de seus mecanismos de ação, são de extrema importância no contexto da odontologia, visto que a exodontia de terceiros molares é um dos procedimentos rotineiros mais realizados por cirurgiões-dentistas e tem sido cada vez mais comum relatos de pós-operatórios desconfortáveis e dolorosos por pacientes submetidos a esse tipo de intervenção cirúrgica (Saluja et al., 2011; Afat et al., 2019).

Da Silva et al., (2021) avaliaram o uso do plasma rico em fibrina em alvéolo após exodontia de terceiros molares inferiores. Ensaio clínico randomizado, com amostra composta por 14 voluntários, divididos em Grupo Controle e Grupo LPRF. Observou-se uma discreta melhora do padrão inflamatório, todavia tal benefício nem sempre fica evidente, sendo necessário mais pesquisas acerca da temática. Daugela et al., (2018) observaram uma promoção no processo de cicatrização, bem como uma cirurgia menos invasiva e um tratamento menos traumático.

Existe uma quantidade significativa entre as pesquisas realizadas e publicadas de trabalhos que retrata de forma positiva o uso deste biomaterial elucidando os benefícios do mesmo no processo de cicatrização de tecidos moles, fato este que ocorre através da sua capacidade de secretar fatores de crescimento a partir do coágulo de fibrina. A integração desta rede de fibrina no sítio regenerativo facilita a migração celular, particularmente para células endoteliais (VEGF) necessárias para a neoangiogênese e as citosinas de plaquetas (PDGF, TGF- $\beta$, IGF- 1) são gradualmente liberadas à medida que a matriz de fibrina é reabsorvida, criando assim um processo perpétuo de cura. Por fim, a presença de leucócitos e citocinas na tela de fibrina, podem desempenhar um papel significativo na autorregulação dos fenômenos inflamatórios e infecciosos (Miron et al., 2017; Fursel et al., 2021).

À medida que ocorre esta autorregulação no sítio cirúrgico, torna-se evidente o potencial hemostático que este biomaterial pode proporcionar, facilitando a prática clínica do cirurgião e tornando a experiência menos invasiva para o paciente.

Pode-se observar que ao longo dos anos, com a inserção de novos protocolos de centrifugação, vêm surgindo cada vez mais novas variações deste biomaterial capazes de amenizar problemas rotineiros derivados de procedimentos cirúrgicos na clínica odontológica. (Bao et al., 2021).

\section{Conclusão}

Concluímos que o uso do L-PRF em cirurgias de terceiros molares é uma alternativa capaz de proporcionar um pósoperatório menos invasivo ao paciente, que está ao alcance do cirurgião-dentista, por apresentar um baixo custo e facilidade de manipulação. Para futuros trabalhos sugerimos uma padronização do acompanhamento utilizado com trabalhos já publicados para uma melhor comparação da eficácia do uso do L-PRF.

\section{Referências}

Afat, I. M., Akdoğan, E. T., \& Gönül, O. (2019). Effects of leukocyte- and platelet-rich fibrin alone and combined with hyaluronic acid on early soft tissue healing after surgical extraction of impacted mandibular third molars: A prospective clinical study. Journal of cranio-maxillo-facial surgery : official publication of the European Association for Cranio-Maxillo-Facial Surgery, 47(2), 280-286.

Bao, M., Du, G., Zhang, Y., Ma, P., Cao, Y., \& Li, C. (2021). Application of Platelet-Rich Fibrin Derivatives for Mandibular Third Molar Extraction Related Post-Operative Sequelae: A Systematic Review and Network Meta-Analysis. Journal of oral and maxillofacial surgery : official journal of the American Association of Oral and Maxillofacial Surgeons, 79(12), 2421-2432.

Benedito, I. de M. V., Góes, R. W. L., Dietrich, L., \& Costa, M. D. M. de A. (2021). Use of PRF for repair of alveole after exodontia of third molars. Research, Society and Development, 10(14), e459101422314.

Campos, L. B., Dietrich, L., Sousa, P. C. S. de, Andrade, C. M. de O., Costa, M. D. M. de A., \& Martins, V. da M. (2021). Fibrina Rica em Plaquetas (PRF) como assistente em Implantologia Oral: relato de caso. Research, Society and Development, 10 (16), e132101623503.

Caymaz, M. G., \& Uyanik, L. O. (2019). Comparison of the effect of advanced platelet-rich fibrin and leukocyte- and platelet-rich fibrin on outcomes after removal of impacted mandibular third molar: A randomized split-mouth study. Nigerian journal of clinical practice, 22(4), 546-552.

Choukroun, J., Diss, A., Simonpieri, A., Girard, M. O., Schoeffler, C., Dohan, S. L., Dohan, A. J., Mouhyi, J., \& Dohan, D. M. (2006). Platelet-rich fibrin (PRF): a second-generation platelet concentrate. Part IV: clinical effects on tissue healing. Oral surgery, oral medicine, oral pathology, oral radiology, and endodontics, 101(3), e56-e60. 
Costa, K. L., Santos, M. de V.., \& Santos, M. D. da S. (2021). Fibrina rica em plaquetas e leucócitos-L-PRF- em Odontologia: revisão da literatura. Research, Society and Development, $10(11)$,

Daugela, P., Grimuta, V., Sakavicius, D., Jonaitis, J., \& Juodzbalys, G. (2018). Influence of leukocyte- and platelet-rich fibrin (L-PRF) on the outcomes of impacted mandibular third molar removal surgery: A split-mouth randomized clinical trial. Quintessence international (Berlin, Germany: 1985), 49(5), 377388.

Dohan, D. M., Choukroun, J., Diss, A., Dohan, S. L., Dohan, A. J., Mouhyi, J., \& Gogly, B. (2006). Platelet-rich fibrin (PRF): a second-generation platelet concentrate. Part I: technological concepts and evolution. Oral surgery, oral medicine, oral pathology, oral radiology, and endodontics, 101(3), e37-e44.

Favero, H. R. Z. (2017). Revisão comparativa entre agregados plaquetários e sangue total relacionados com osseointegração e titânio. Trabalho de Conclusão de Curso, Universidade Federal de Santa Catarina, SC. https://repositorio.ufsc.br/handle/123456789/181325

Fursel, K. de A., Oliveira Neto, JL de., Sousa, MJ de., Moreira, VHL de O.., \& Silveira, RJ. (2021). Propriedades da fibrina rica em plaquetas (PRF) aplicada à cirurgia oral - protocolo de Choukroun. Pesquisa, Sociedade e Desenvolviment, 10 (5), e59510515338.

Gonçalves, S. A., Teodoro, T. A. D., Oliveira, Érika T. de., Andrade, C. M. de O., Dietrich, L., \& Costa, M. D. M. de A. (2021). Comparison between the conventional technique after third molar extraction and the use of fibrin-rich plasma associated with ozone therapy: case report. Research, Society and Development, 10(15), e180101522881.

Miron, R. J., Fujioka-Kobayashi, M., Bishara, M., Zhang, Y., Hernandez, M., \& Choukroun, J. (2017). Platelet-Rich Fibrin and Soft Tissue Wound Healing: A Systematic Review. Tissue engineering. Part B, Reviews, 23(1), 83-99. https://doi.org/10.1089/ten.TEB.2016.0233

Mourão, C. F. A. B., Valiense, H., Melo, E. R., Mourão, N. B, M, F, \& Maia, M. D. C. (2015). Obtenção da fibrina rica em plaquetas injetável (i-PRF) e sua polimerização com enxerto ósseo: nota técnica. Rev. Col. Bras. Cir. 42(6), 421-423.

Peralvo, A. O. S., García A. S., \& Fluente L. A. (2017). Nuevas tendencias en regeneración tisular: fibrina rica en plaquetas y leucocitos. Revista Española de Cirugía Oral y Maxilofacial, 39(2), 91-98.

Pereira, A., Shitsuka, D., Parreira, F., \& Shitsuka, R. (2018). Metodologia da pesquisa científica [free e-book]. Santa Maria/RS. Ed. UAB/NTE/UFSM.

Saluja,H., Dehane,V. \& Mahindra,U. (2011). PlateletRich fibrin: Platelet-Rich fibrin: A second generation platelet concentrate and a new friend of oral and maxillofacial surgeons. Ann Maxillofac Surg., 1: 53-57.

Santos Gonçalves, R. M., Conceição, V. S., Bezerra, R. M., de Almeida Junior, V. R., Teixeira Cangussu, M. C., \& Santana Sardinha, S. de C. (2020). Aplicação da fibrina rica em plaquetas em cirurgia de terceiros molares: avaliação do sangramento (parte I) Revista Da Faculdade De Odontologia Da Universidade Federal Da Bahia, 50(1), 15-22

Silva Conceição, V., Mício Santos Gonçalves, R., Macedo Bezerra, R., Rodrigues de Almeida Junior, V., Cristina Teixeira Cangussu, M., \& de Cássia Santana Sardinha, S. (2020). Aplicação da fibrina rica em plaquetas em cirurgia de terceiros molares: avaliação da dor (parte II) Revista Da Faculdade De Odontologia Da Universidade Federal Da Bahia, 50(2).

Uyanık, L. O., Bilginaylar, K., \& Etikan, İ. (2015). Effects of platelet-rich fibrin and piezosurgery on impacted mandibular third molar surgery outcomes. Head \& face medicine, 11, 25. 